\title{
Evaluación del cambio de uso de suelo en el parque nacional "La Marquesa" (1994-2007), aplicando tecnología SIG
}

\author{
Mónica Chico Avelino, Miguel Ángel Trinidad \\ Trinidad y Raymundo Montoya Ayala
}

\section{Resumen}

El Parque Nacional Insurgente Miguel Hidalgo y Costilla, conocido como "La Marquesa", fue decretado en 1936 como Área Natural Protegida. A pesar de su condición de protección y conservación ha sufrido modificaciones en la cobertura vegetal, lo que ha generado problemáticas en sus ámbitos eco sistémicos. Por ello, el presente trabajo tuvo como objetivo evaluar los cambios de uso de suelo del año 1994 al 2007, con el fin de establecer una propuesta orientada a la conservación de esta Área Natural Protegida y la preservación de sus características ecológicas. Para cumplir con el objetivo se emplearon ortofotos correspondientes al periodo 1994, 1999 y 2007 a escala 1:75,000 para la digitalización de los distintos tipos de vegetación y usos del suelo logrando definir once categorías. Los resultados evidenciaron que el área ha experimentado modificaciones en la dinámica de utilización del territorio, siendo las actividades agrícolas y la mancha urbana, sobre todo para las actividades recreativas y de turismo que han tenido la mayor expansión, favoreciendo la deforestación y el crecimiento de los pastizales como consecuencia de la ganadería mal planeada, lo cual conlleva al disminución de la recarga de acuíferos y un gran impacto en los sistemas ambientales, por falta de políticas de manejo. De igual manera se logró identificar que entre las temporalidades de 1999 y 2007 es donde se presentaron los cambios más evidentes.

Palabras clave: Cambio de uso de suelo. La Marquesa. SIG. Mancha urbana. Gestión territorial.

\section{Abstract}

The National Park Miguel Hidalgo y Costilla, commonly known as "La Marquesa", was established in 1936 as a Natural Protected Area. In spite of the status as a protected area, its vegetative cover has suffered considerable modifications during the last decades, resulting in severe ecosystemic problems. This paper aims, firstly, to describe land-use changes between 1994 to 2007. Secondly, on the basis of this 
diachronic description, we will propose conservation strategies in order to better protect the national park and its ecological characteristics. Orthophotographs of 1994, 1999, and 2007 at the 1:75000 scale have been used to digitize and analyze types of vegetation over the sample period. Based on this analysis, we defined eleven categories of land use. The results demonstrate that the area has undergone changes regarding the dynamics of land use, for example with respect to agricultural activities and urban sprawl. Expansion of touristic and recreational facilities caused deforestation, while poorly planned livestock farming resulted in pasture growth, which in turn led to decreasing groundwater resources and, therefore, had a major impact on environmental systems. These transformations are particularly apparent for the period 1999-2007.

Keywords: Land-use changes. La Marquesa. GIS. Urban growth. Regional planning.

Mónica Chico Avelino (vocesantiguas@hotmail.com); Miguel Ángel Trinidad Trinidad (principe_maquiavelo@comunidad.unam.mx); Raymundo Montoya Ayala (Icazallas@terra.es). Facultad de Estudios Superiores Iztacala, Universidad Nacional Autónoma de México, Laboratorio de SIG y Análisis Espacial, Unidad de Biotecnología y Prototipos. 


\section{INTRODUCCIÓN}

En las últimas décadas el cambio del uso de suelo se ha constituido como uno de los factores plenamente implicados en el cambio global, que ha generado alteración en ciertos procesos y ciclos. Lo anterior se vuelve trascendental si se considera que es a través de estos cambios en donde se hace evidente la relación entre el hombre y su entorno (Lambin et al., 1999). Los ecosistemas terrestres han sufrido grandes transformaciones, la mayoría debido a la conversión de la cobertura del terreno y a la degradación e intensificación del uso del suelo (Lambin, 1997).

Esta dinámica del uso de suelo, tiende al reemplazo de paisajes naturales por tierras agrícolas y urbanas, lo cual repercute negativamente en la diversidad, coherencia, identidad y en los servicios ambientales que los sistemas naturales ofrecen (Antrop, 2005).

El fenómeno de cambio de uso de suelo, ha sido muy acelerado lo que ha llevado a la pérdida sobre todo de la cubierta forestal, que trae consigo el agotamiento de la biodiversidad. De igual manera el potencial de uso de los múltiples bienes y servicios ambientales que proporcionan los ecosistemas para el bienestar humano, así como el calentamiento global, alteración de ciclos hidrológicos y biogeoquímicos, la introducción de especies exóticas, desplazamiento de especies nativas y la pérdida de hábitats (Velázquez et al., 2002).

La deforestación y el uso del suelo en México se encuentran muy relacionados siendo problemáticas que se han presentado desde tiempos precolombinos, sin embargo, durante las últimas cinco décadas estos procesos han incrementado dramáticamente, con un panorama poco alentador, las 52 millones de hectáreas de bosques y selvas con que contaba el país en el año 2000, presentaron una tasa de deforestación promedio de 631 mil ha/año (FAO, 2005).

Bajo este contexto en donde las actividades humanas han sido determinantes resultando en efectos como la degradación ambiental, debido a que los usos de suelo son diversos y varían en intensidad, duración y extensión, que repercuten en la sustentabilidad de los sistemas y por lo tanto en la calidad de vida de la población. Es por eso que la evaluación y análisis de los cambios de uso del suelo como factor territorial son fundamentales para entender cómo, dónde y qué tanto se están perdiendo los recursos naturales, que contribuya a la toma de decisiones que contribuyan en los ámbitos ecológicos y sociales.

El Parque Nacional Insurgente Miguel Hidalgo y Costilla, fue decretado en 
1936 como Área Natural Protegida (ANP) (SEMARNAT, 2010), el parque se localiza en la región central de México en el Valle de México, dentro del Sistema Volcánico Transversal (ver Mapa 1-A) en el centro del Estado de México y al occidente del DF (Mapa 1-B) comprendiendo tres municipios Lerma, Ocoyoacac y Huixquilucan, en el Estado de México, y la Delegación Cuajimalpa en el D.F. (Mapa 1-C). Tiene una extensión de 1,760 ha, una altitud que va de los 2,400 a 3,100 msnm.

El área cuenta con una superficie boscosa del 70\%, dentro de la cual se hallan importantes elevaciones. El resto de la superficie del parque está conformada por lomeríos, llanos, claros boscosos y la Laguna de Salazar. Dentro de estas áreas forestales se localiza infraestructura de recreación como una estación piscícola y diversos espacios dedicados al servicio de alimentos y bebidas, renta de caballos y cuatrimotos, que se encuentran próximas a la carretera México-Toluca. Este sitio es la parte más conocida y visitada de todo el ANP a la que popularmente se conoce como "La Marquesa".

Mapa1. Localización del Parque Nacional Insurgente Miguel Hidalgo y Costilla
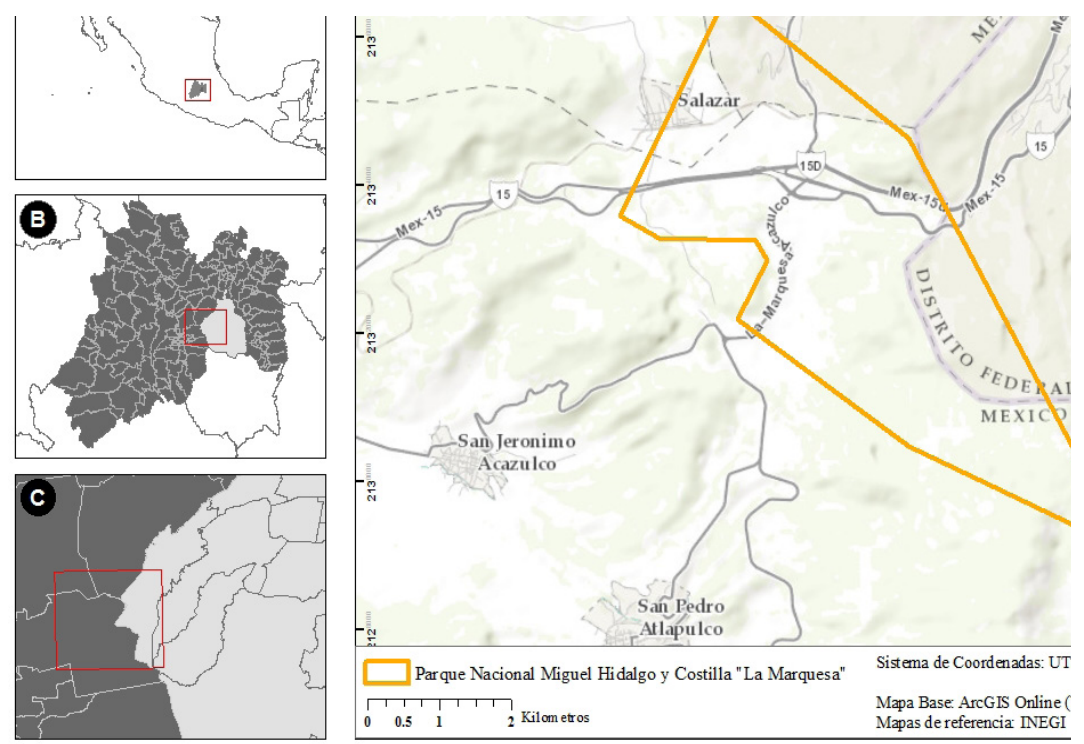

Fuente: ArcGis Online (World Topographic Map) e INEGI

A pesar de contar con las características físicas y ambientales para cumplir con las funciones de investigación, educación y recreación, presenta una serie de problemas administrativos derivados de su condición como ANP y además por su 
gran actividad recreativa y turística, por lo que ha sufrido de una fuerte presión ambiental. Por ello este análisis determina los cambios de uso de suelo de 1994 al 2007 para establecer una propuesta orientada a la conservación de esta área natural protegida y la preservación de sus características ecológicas.

\section{METODOLOGÍA}

Se recopilo la información hasta el momento existente del área, como cartografía base de hidrología, geología, vegetación, edafología, a escala 1: 250000 de Instituto Nacional de Estadística Geografía (INEGI), con el fin de caracterizar el área.

Para la evaluación del cambio del uso del suelo se realizó la digitalización de las distintas clases de uso del suelo del parque, la cual se basó en la identificación de la variedad de coberturas en el territorio, en las temporalidades de 1994, 1999 y 2007, lo cual permitió expresar las diferencias entre tres momentos para las unidades de observación.

Se utilizaron ortofotos basadas en las fotografías aéreas escala 1:75,000 del mes de abril para cada uno de los años, en proyección Universal Transversa de Mercator (UTM), zona 14Q, con resolución espacial de $2 \mathrm{~m}$, para el caso de las ortofotos del año 1994 se encontraban referidas al Datum NAD27 y al elipsoide Clarke 1866, mientras que las de los años 1999 y 2007 al Datum ITRF92 y al elipsoide GRS 80, a las primeras se les realizó un cambio en la proyección, con el fin de tener la información en el mismo sistema de referencia. Una vez definidas las clases, se procedió al análisis del cambio en los usos de suelo de las tres temporalidades, mediante los las pérdidas y ganancias en hectáreas de las clases definidas.

\section{RESULTADOS Y ANÁLISIS}

El método a partir de la digitalización permitió la determinación y clasificación de 12 categorías de vegetación y usos de suelo, que se pueden observar en el Mapa 2 de los usos de suelo correspondientes a las tres temporalidades. 
Mapa 2. Categorías de uso de suelo para el año1994, 1999 y 2007 del ANP
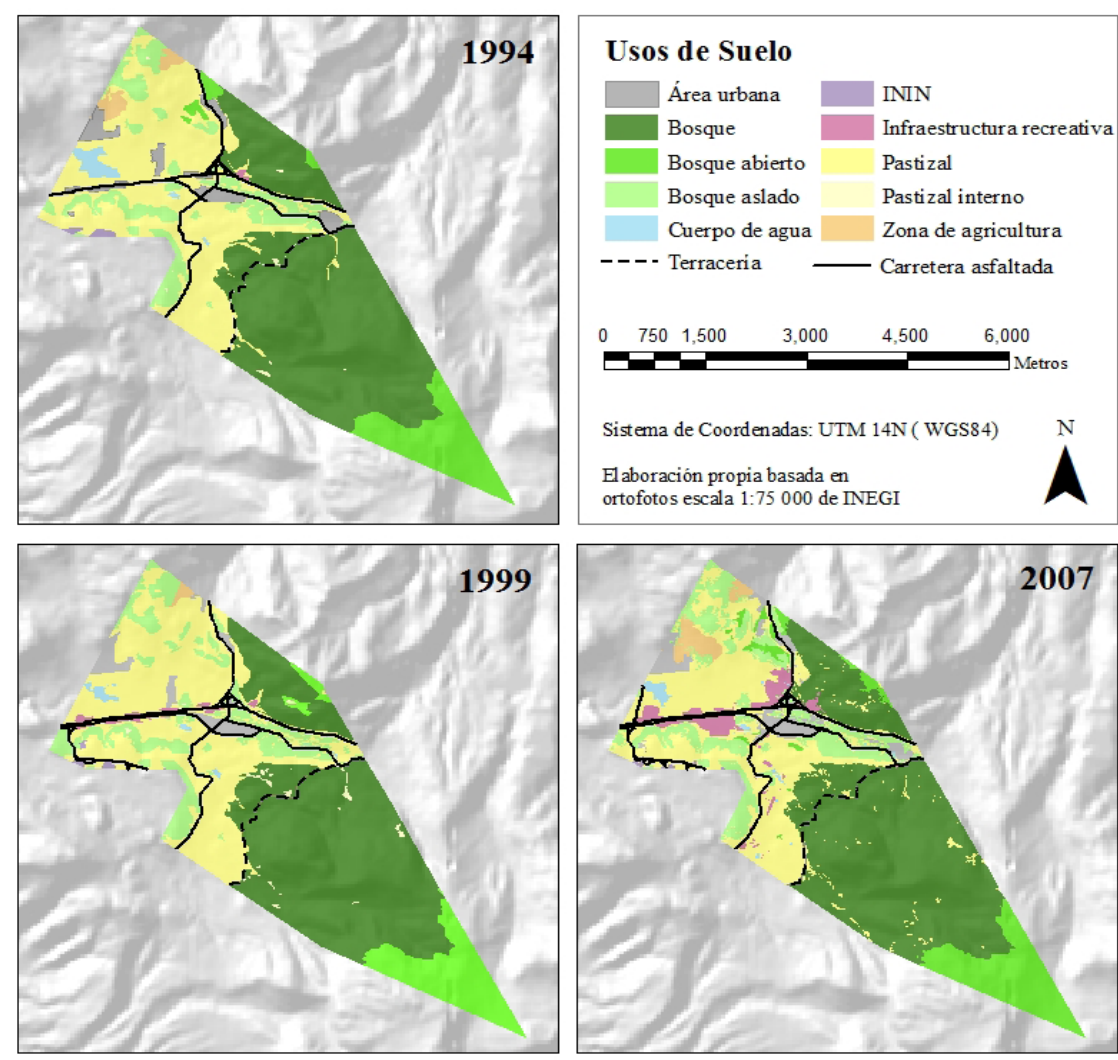

Fuente: Elaboración propia.

La sobre posición de los polígonos evidenció que no obstante su condición como ANP, ha experimentado modificaciones en los patrones de uso de suelo, siendo las actividades agrícolas y principalmente la mancha urbana (incluyendo aquí las actividades recreativas y el turismo) que han tenido mayor expansión, mientras que las zonas forestales han sufrido una dinámica contraria presentando procesos de fragmentación, por otro lado el cuerpo de agua inmerso en el ANP, la laguna Salazar disminuyó su superficie (Figura 1). 
Figura 1. Cambio de uso de suelo de 1994 al 2007, de las 11 categorías de uso definidas

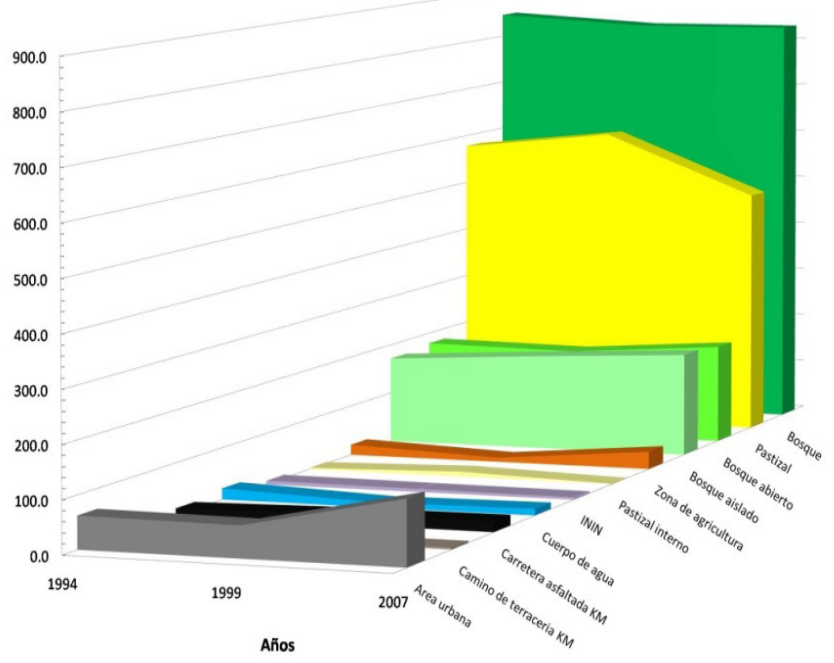

Fuente: Elaboración propia.

El bosque de Abies es la vegetación dominante en el parque, el cual se encuentra principalmente en lugares protegidos, como son cumbres y laderas de montañas, cañadas o barrancas. Este tipo de vegetación se presenta en un intervalo altitudinal que va de los 2,600 a 3,660 msnm. Esta cobertura forestal es motivo la declaratoria de la zona como ANP, debido a su importancia biológica, ecológica, cultura y social. El principal atractivo del bosque es su belleza escénica y natural con vegetación original y abundante, por lo que cuenta con grandes servicios para la recreación y el esparcimiento de sus visitantes.

Sin embargo, a pesar de su gran importancia se ha enfrentado a diversas problemáticas como la tala clandestina de arbolado, la explotación de los recursos de manera descontrolada, la ganadería y la agricultura intensiva, así como el constante crecimiento de comercios, ganando superficie a la vegetación de tipo forestal. Es así que el cambio en la cobertura del bosque a lo largo de 13 años (Figura 2a) se ha visto afectado, reduciendo su distribución. El análisis de las imágenes de 1994 a 1999 resalta que se tiene una pérdida del $2 \%$ en su cobertura, y de $9 \%$ del 1994 al 2007. El cambio de este tipo de vegetación a otros usos de tipo antrópico ha generado una evidente fragmentación, lo que implica una gran vulnerabilidad de las especies nativas, ya que al aumentar la fragmentación aumenta el efecto 
borde generando mayor presión a los manchones que quedan aislados, provocando la pérdida de la cobertura que alberga especies de importancia ecológica. Es importante hacer notar que del año 2000 a la fecha se han hecho programas de reforestación del bosque (SAGARPA, 2011), como parte de algunos esfuerzos de conservación de los espacios protegidos, contrariamente a estas estrategias el Estado de México en el 2007 se encontraba dentro de los cinco estados con más deforestación en todo el país (Reyes et al., 2003). Es decir existe una fuerte dinámica negativa incluso al contar con programas de restauración no se alcanzan los objetivos debido al incumplimiento de políticas que se encuentran asociadas a la gestión de estas áreas.

Otro punto importante radica en determinar cuáles son los factores socioeconómicos que más inciden en los procesos de deforestación, de los cuales algunos estudios consideran como principal causa los aspectos demográficos (Lambin, 1997). En la comunidad de La Marquesa se contaba con 692 habitantes en 1995 (INEGI, 1995), mientras que para el 2010 (INEGI, 2010) el censo reporto 981 habitantes, esto significa que el crecimiento demográfico es mayor al 40\%, lo cual explicaría la pérdida en la cobertura del bosque.

El avance demográfico afecta significativamente al bosque y a cualquier tipo de vegetación. Este concepto va de la mano con los siguientes usos de suelo reportados en La Marquesa, como el bosque abierto (Figura 2c), el cual disminuyó su cobertura en el periodo de 1994 a 1999 (5 años) tiene -3\% de cobertura; sin embargo para el periodo de 1999 a 2007 (8 años) aumentó hasta un 12\% su distribución espacial. Probablemente se deba a la tala clandestina, lo que ocasiona básicamente formaciones con una distribución discontinua de árboles (fragmentación). Además la actividad agrícola que incrementa la distribución y superficie en el área, debido a que en el parque se han realizado desmontes, para introducir sistemas agrarios y usos de tipo turístico, para satisfacer las necesidades de los visitantes.

El caso contrario pasa con el bosque aislado (Figura 2b), el cual en 1994 tenía 171.5 ha y para el 1999 aumentó un 10\% contando con 188.9 ha, mientras que, de las 188.9 Ha que comprendía en 1999, para el 2007 tenía 203.6 ha, lo que significa un aumento de cobertura del $8 \%$. Estos resultados tienen dos vertientes; por una parte los esfuerzos para contrarrestar los efectos por cambio de uso de suelo como son los programas de reforestación que han favorecido hasta cierto punto a la cobertura del bosque pero que presenta un patrón de plantación aislada. Por otro lado aquellos de tipo antrópico como el aumento de la actividad agrícola, en la zonas de vegetación forestal; en ambos casos son principalmente los pastizales quienes seden sus áreas y en consecuencia lo que ocasiona estas nuevas islas 
de bosque, localizadas principalmente en las áreas cercanas a Huixquilucan. Por lo que si esta dinámica fragmentaria permanece puede derivar en la discontinuidad de la vegetación, y por lo tanto el desplazamiento de especies nativas por especies exóticas y deterioro en los servicios eco sistémicos. En tanto a las acciones que se han tomado para conservar y restaurar el parque, como son la reforestación, estas mejoran la calidad de vida de los habitantes sin depredar o exceder el consumo de los recursos, ya que son la fuente básica del turismo del lugar, es por eso que sería conveniente establecer estrategias de turismo alternativo que no involucren procesos de pérdida de cobertura vegetal y por lo tanto de biodiversidad, ya que este fenómeno pone en riesgo la categoría del ANP, al no cumplir con los objetivos de su categoría que son como Parque Nacional la conservación de la flora y de la fauna, y de las aguas del área; además de potencializar su valor científico, histórico escénico, educativo, de recreo y su aptitud para el desarrollo del turismo, sin comprometer su sustentabilidad.

Figura 2. Cambios del bosque (a), bosque aislado (b) y bosque abierto (c) en 13 años $(94,99,07)$

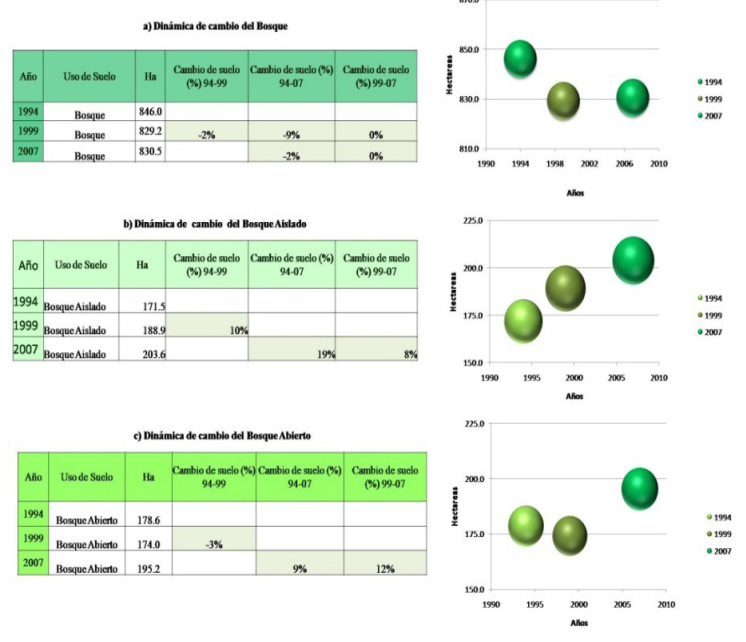

Fuente: Elaboración propia.

Esta tendencia indica que de manera constante el bosque en general se ha visto vulnerable ante los proceso de fragmentación, ya que los bosques aislados y abiertos han ganado superficie como se puede apreciar en la Figura 2 y en el Mapa 1 , siendo remplazados por usos como son pastizales en el interior de los bosques e infraestructura recreativa principalmente. Esto indica que se requiere de un esfuerzo más dedicado para combatir la tala clandestina, las actividades agrícolas y 
sobre todo el crecimiento urbano dentro del parque, que generan una fuerte presión ambiental en estos paisajes.

Por su parte el pastizal ha tenido pérdidas en su cobertura sobre todo en el intervalo de 1999 al 2007 (Figura 3a). En 1994 contaba con 579.9 ha y en 13 años perdió el 21\% de su distribución contemplando hasta el 2007 con 490.3 ha, esto debido a la gran expansión urbana, sobre todo del tipo recreativo (turístico), el cual ha resultado en una mayor demanda de agua, y esto aunado a la reducción de los sistemas forestales ha disminuido la recarga de los mantos acuíferos por lo que la Laguna Salazar ha experimentado una dinámica bastante severa de reducción en su superficie.

Como ya se mencionó la población es un eje directriz en la dinámica de la utilización del suelo, es así como las actividades agrícolas han perturbado gran parte del bosque, lo que ha ocasionado modificaciones en el hábitat. Las zonas agrícolas (Figura 3b) han aumentado en 13 años un $70 \%$ en su cobertura, mientras que la zona urbana (Figura 3c) han incrementado en un $98 \%$ su plaza.

Figura 3. Cambios en 13 años de pastizal (a), zonas agrícolas (b), áreas urbanas (c)en donde se muestran las hectáreas siendo del 99 al 2007 el intervalo de tiempo con mayores modificaciones


Fuente: Elaboración propia. 
Los factores urbanístico y agrícola, tienen un comportamiento similar, ya que de 1994 a 1999 la agricultura disminuyó un 59\%, de 1999 a 2007 la agricultura aumentó más del $300 \%$, en tanto que la zona urbana está enfocada principalmente a la creación de infraestructura de servicios turísticos y recreativos también tuvo un ascenso del 106\%, es decir, el crecimiento de estas actividades de esparcimiento han ganado superficie sobre los pastizales e incluso el bosque.

La superficie del bosque, ante los escenarios de fragmentación, ha presentando una dinámica fuerte ya que contaba para el año 1994 con 486 ha, mostrando una distribución espacial con poca segmentación, sin embargo en el año 1999 esta superficie cambio a 829 ha, pasando a usos de suelo del tipo antrópico como son áreas urbanas, infraestructura (recreativa, terracerías y carreteras ), pastizal (interno) y a la propia fragmentación del bosque (abierto y aislado), que fue la que mayor aumento en superficie evidencio, mientras que para el año 2007, aumentó 1 ha y cambió a las clases de uso del mismo orden sólo que en este caso el pastizal fue el que mayor cobertura ganó.(Figura 4).

Figura 4. Cambios del bosque en las 3 temporalidades representado en porcentaje, mostrando los usos en los que ha derivado, siendo el bosque fragmentado y el pastizal los que más superficie han ganado

1994

1999

2007

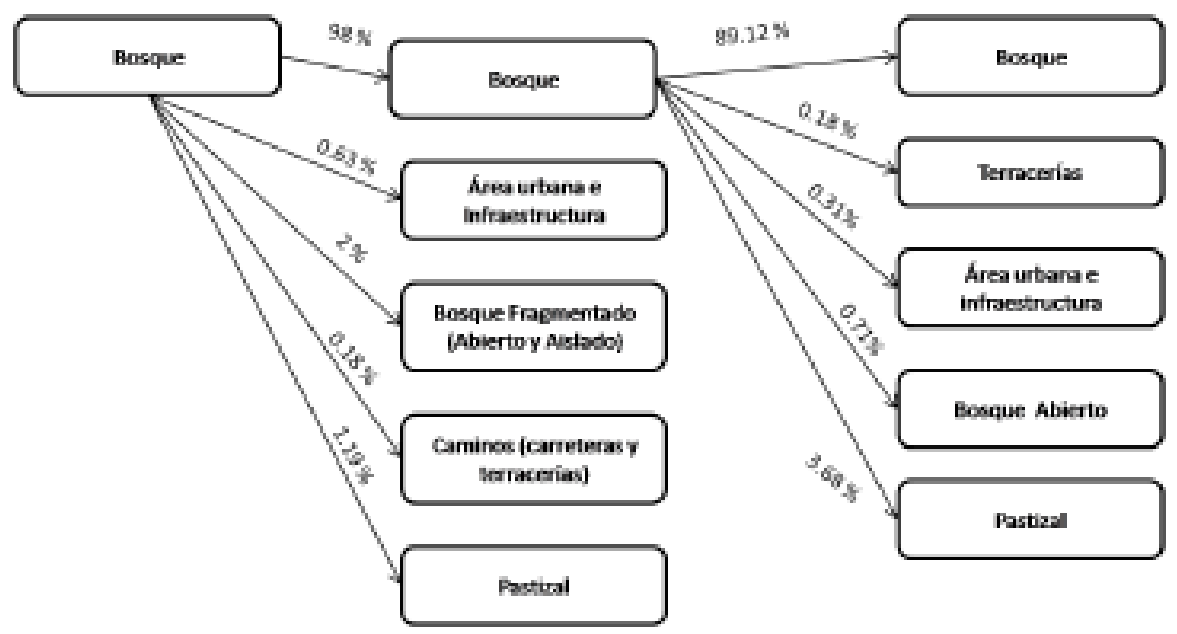

Fuente: Elaboración propia. 
Por su parte la mancha urbana ha crecido considerablemente en el área, sobre todo para satisfacer la demanda por parte de las actividades turísticas, siendo también el pastizal el que ha sido susceptible a ser cambiado a usos urbanos, ya que para el pastizal en el año 1994 comprendía 579 ha de superficie mientras que para el 1999 esta disminuyó el 10\%, siendo transformado a usos como son agricultura, INNIN, cuerpos de agua, pastizal interno, bosque aislado, área urbana, infraestructura recreativa y caminos (terracerías y asfaltadas), para el año 2007 disminuyó en un $20 \%$, mientras que las zonas agrícolas aumentan su cobertura $4 \%$; la zona urbana, la infraestructura recreativa y los caminos incrementaron su superficie en conjunto en un 10\%, siendo la infraestructura la que gano más área con un $8 \%$. (Figura 5 ).

Figura 5. Cambios de pastizal en las 3 temporalidades representado en porcentaje de los cambios de uso, la que mayor superficie gano fueron las áreas urbanas

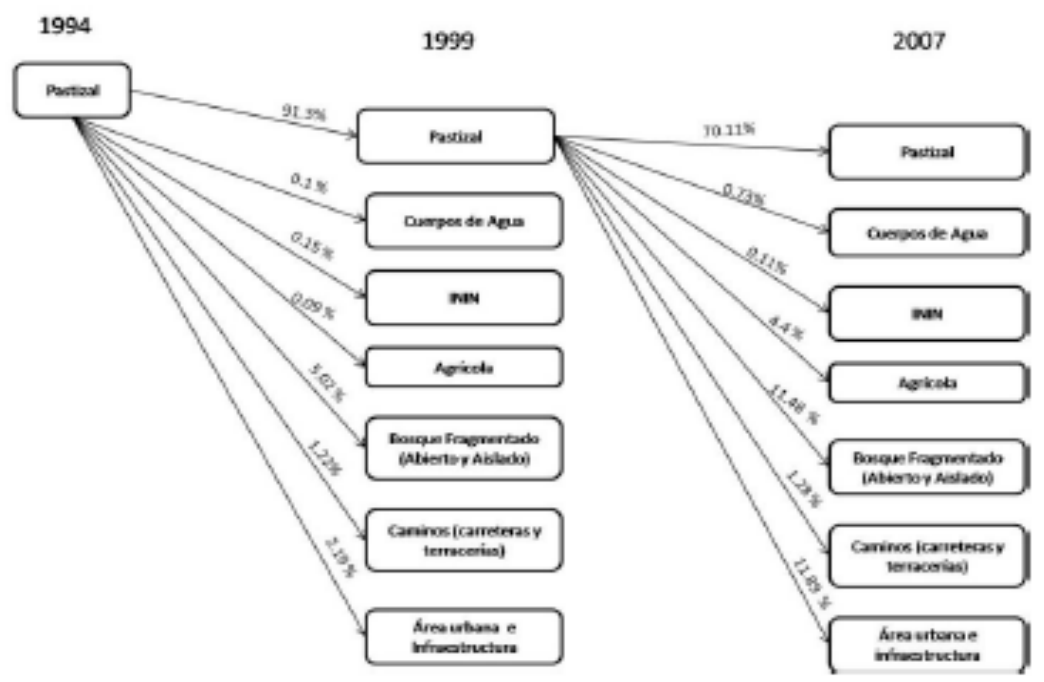

Fuente: Elaboración propia.

Esta dinámica tan acelerada de los espacios urbanos trae consigo cambios significativos en la vegetación, en este caso, la infraestructura vial (Figura 6) es la que ha tenido un aumento importante debido a que mientras más cobertura presenta las zonas de servicios recreativos y turísticos, mayor necesidad de comunicación entre estos espacios se requiere. Como se observa en el Mapa 1, en 1994 el área no contaba con una red viaria tan compleja como la que se presenta en el año 2007, en este momento incluso las terracerías se conectan con las carreteras asfaltadas y estas últimas aumentan considerablemente. En 1994 había $13.7 \mathrm{~km}$ destinadas a carreteras asfaltadas y $3.1 \mathrm{~km}$ de terracería, igualmente, en el 2007 
se incrementó en un $47 \%$ la superficie ocupada por las carreteras asfaltadas con $20.1 \mathrm{~km}$ y un $7 \%$ los caminos de terracería con $3.3 \mathrm{~km}$.

Figura 6. Distribución espacial de las vías de comunicación a lo largo de 13 años

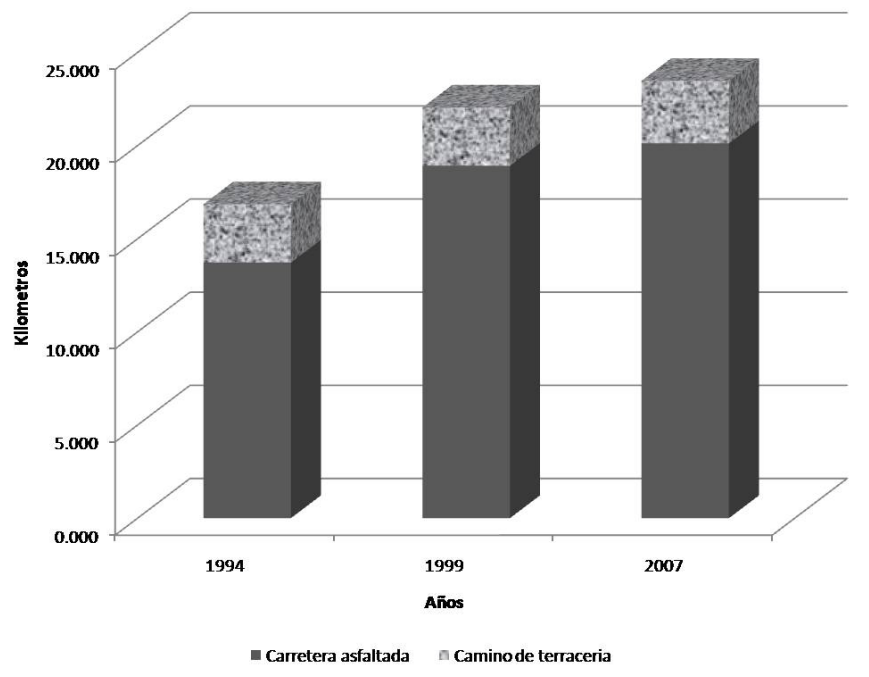

Fuente: Elaboración propia.

Es así como las vías de comunicación también afectan directamente las zonas boscosas y de pastizales, disminuyendo la calidad de la vegetación del parque, que son el hábitat de especies endémicas y en peligro de extinción como el conejo zacatuche y el gorrión serrano.

Otro uso de suelo muy importante son los cuerpos de agua que alberga el parque, ya que aporta de sus aguas a la Ciudad de México; vierte sus aguas a la cuenca del Río Lerma y al Río Amacuzac, que forma parte de la cuenca media del Río Balsas. A lo largo de 13 años estos cuerpos de agua (Figura 7) se han visto afectados por la influencia humana principalmente. En 1994 había 21.4 ha, mientras que para el 2007 solo habían 12.4 ha, lo que significa una pérdida del 42\%. 
Figura 7. Cambios de los cuerpos de agua, en donde las pérdidas mayores se evidencian en el periodo del 1994 al 1999, reduciendo su superficie en un 55\%
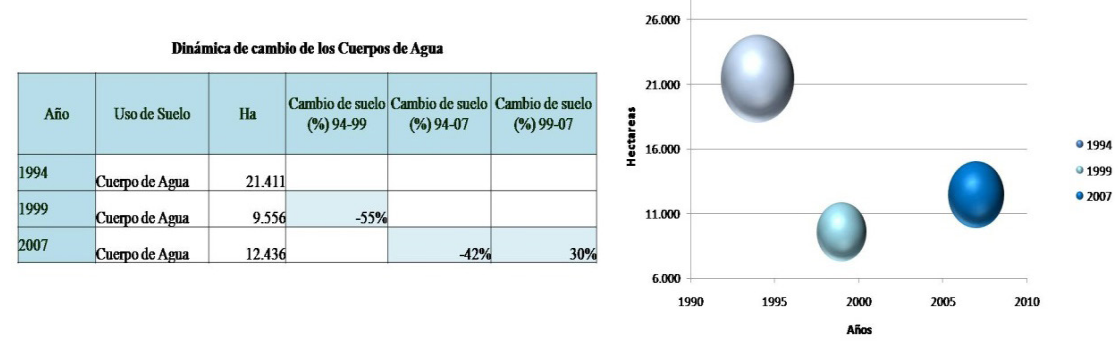

Fuente: Elaboración propia.

El área que abarca el Instituto Nacional de Investigaciones Nucleares (ININ) que tiene parte de sus instalaciones en las inmediaciones e incluso dentro del parque, no ha tenido repercusiones significativas sobre el área, ya que tiene vallada sus instalaciones e incluso protege el bosque que mantiene en su interior; al contrario de las actividades humanas, el ININ tiene acciones de reforestación, en el 2011 desarrollaron una campaña en donde se plantaron un total de 3,500 árboles (ININ, 2011).

\section{CONCLUSIONES}

El Parque Nacional "La marquesa", a pesar de albergar diversos sistemas naturales, que se encuentran bajo la categoría de protección, ha sufrido diversas modificaciones en los usos de suelo, siendo las actividades agrícolas y principalmente la mancha urbana, sobre todo las actividades recreativas y el turismo que han tenido mayor expansión e impacto, los principales cambios que se identificaron se dieron en el intervalo del año 1999 a 2007.

Los sistemas forestales han sido los más vulnerables ante los patrones de utilización del territorio, generando así un escenario de fragmentación de la vegetación natural, aumentando el efecto borde que puede derivarse en el desplazamiento de especies nativas frente a especie exóticas, de fragilidad del paisaje, además de un decremento en los servicios ambientales del área como la captura de carbono, y la recarga de acuíferos. Es así como el crecimiento demográfico ha derivado en el aumento de los bosques abiertos, que se atribuye a la tala, que genera los manchones de esta vegetación. 
Las zonas agrícolas han aumentado en 13 años su cobertura de manera significativa, un $70 \%$ de su superficie del tiempo inicial tomado para este trabajo. Sin embargo el uso que ha presentado una dinámica mucho mayor y acelerada es el urbano que ha incrementado en un $98 \%$ su ocupación en el territorio, generando una intensa presión a los sistemas forestales principalmente.

Además este crecimiento urbano sobre todo la infraestructura recreativa para satisfacer las demandas de las actividades turísticas del área, ha tenido como resultado mayor demanda del recurso agua, este patrón de consumo, ha derivado en una sobreexplotación de los acuíferos, que a su vez ha disminuido la capacidad de recarga de estos mantos acuíferos por la reducción del tamaño del bosque.

En la zona se han implementado diversas estrategias de conservación, como campañas de reforestación de ciertas zonas boscosas del área, que permitieron una recuperación de la cobertura vegetal, sin embargo esto también contribuyó al incremento de las zonas urbanas, ya que dentro los principales atractivos de este tipo de espacios son la vegetación y la fauna que alberga que ofrece a los visitantes una interacción con la naturaleza, es por eso que la dinámica de los sistemas forestales se encuentra determinado por la actividades turísticas del sitio. Bajo este panorama, sería importante además del desarrollo de prácticas de conservación, establecer políticas de manejo que permitan una mejor gestión del territorio que permita por un lado satisfacer necesidades sociales y recreativas sin comprometer a los sistemas ambientales.

\section{BIBLIOGRAFÍA}

- Antrop, M. (2005): "Why landscapes of the past are important for the future", in Landscape and urban planning, 2005, No. 70, pp. 21-34.

- FAO (2005). "Forest Resource Assessment" Disponible en: http:www.fao.org.

- Instituto Nacional de Estadística y Geografía (INEGI) (2010). "Censo de Población y Vivienda 2010, Estadística > Censos y Conteos de Población y Vivienda" Disponible en: http://www3.inegi.org.mx.

- Instituto Nacional de Estadística y Geografía (INEGI). (1995). "Conteo de Población y Vivienda 1995, Estadística > Censos y Conteos de Población y Vivienda" Disponible en: http://www3.inegi.org.mx. 
- Instituto Nacional de Investigaciones Nucleares (ININ) (2011). Inicia en el ININ el Programa Reforestación PROÁRBOL en el Sector Energía. México: PROALBOL.

- Lambin, E. F. (1997). Modeling deforestation processes: a review tropical ecosystem environment observation by satellites. Luxembourg: European Commission Joint Research Centre- Institute for Remote Sensing Applications. European Space Agency.

- Lambin, E. F., N; Baulies, G; Bockstael, T; Fisher, R; Krug, E. F; Lemmans, R. R; Moran, Y; Rindfuss, D; Sato, B. L; Skole, Turner II and C. Vogel. (1999). Land use and land cover change implementation strateg. Estocolmo: Comité Científico de Dirección y la Oficina de Proyectos Internacionales de LUCC.

- Reyes, H. H; Cortina, S; Perales, H; Kauffer, E. y Pat-Fernández, J. M. (2003). "Efecto de los subsidios agropecuarios y apoyos gubernamentales sobre la deforestación durante el periodo 1990-2000 en la región de Calakmul, Campeche", en Investigaciones geográficas, 2003, №. 15, pp. 88-106.

- Secretaría de Medio Ambiente y Recursos Naturales (SEMARNAT) (2010). Áreas naturales protegidas. México: SEMARNAT.

- Secretaría de Agricultura, Ganadería, Desarrollo Rural, Pesca y Alimentación (SAGARPA) (2011). "Desarrollo de proyectos para reducir emisiones por degradación de suelos y deforestación, SAGARPA> sala de prensa>" Boletines. Disponible en: http://www.sagarpa.gob.mx/saladeprensa/boletines.

- Velázquez, A; J.F. Mas; J.R. Díaz-Gallegos; R. Mayorga-Saucedo; P.C. Alcántara; R. Castro; T. Fernández; G. Bocco y J.L. Palacio (2002) "Patrones y tasas de cambio del uso del suelo en México", en Gaceta ecológica, 2002, №. 62, pp. 21-37. 